\title{
Bilateral or Unilateral Cross-Bite Treatment with Nickel Titanium Palatal Expander (NPE-2) in Young Adults
}

\author{
Graziano Montaruli ${ }^{1}$, Michele Laurenziello ${ }^{1 *}$, Lucio Lo Russo ${ }^{1}$, Claudio Chimenti², Lorenzo Lo \\ Muzio $^{1}$ and Domenico Ciavarella ${ }^{1}$ \\ ${ }^{1}$ Department of Clinical and Experimental Medicine, School of Dentistry, University of Foggia, Italy \\ ${ }^{2}$ Department of Surgery Sciences, Faculty of Medicine, School of Dentistry, University of L'Aquila, Italy
}

Received: February 23, 2016; Accepted: March 21, 2016; Published: May 19, 2016

*Corresponding author: Michele Laurenziello, Via Rovelli, n. 48, Foggia (FG) 71122, Italy, Tel: +3890287888, E-mail: dr.mlaurenziello@gmail.com

\begin{abstract}
Introduction: The aim of this study was to evaluate skeletal and dento-alveolar effects that the Nickel-Titanium Palatal Expander (NPE-2) allows to obtain on the transverse plane, verifying its effectiveness on permanent dentition in adult patients during the treatment of bilateral or unilateral posterior cross bite.

Methods: 21 patients ( 13 females and 8 males, mean age 17.2 years) in permanent dentition with reduction of the transverse diameter of the maxilla, bilateral or unilateral posterior cross bite, were treated with nickel titanium palatal expander. The posteroanterior radiographic evaluation screening and analysis of casts at the beginning of treatment (T1) and at six months after the application of the device (T2) were performed to study the variations obtained with the NPE-2.

Results: The increase of $2.9 \mathrm{~mm}(P<0.01)$ of the distance between the points $\mathrm{CVM}+\mathrm{d}$ and $\mathrm{CVM}+\mathrm{s}$ valued on the posteroanterior radiograph, the increase of $2.3 \mathrm{~mm}(P<0.01)$ of the upper intermolar width and of $1.6 \mathrm{~mm}(P<0.01)$ of the upper interpremolar width were obtained. No statistically significant increase of the arch perimeter was obtained on the upper arch. No statistically significant increase of the transverse diameters was obtained on the lower arch.

Conclusions: The dentoalveolar changes induced by the application of NPE-2 and the correction of bilateral or unilateral cross-bite showed the effectiveness of this device even in adults in permanent dentition.
\end{abstract}

Keywords: Permenent dentition; Posterior cross bite; Nickeltitanium alloy; Adult orthodontics; Dentoalveolar changes;

\section{Introduction}

The prevalence of Cross Bite (CB) in the primary and mixed dentition within Caucasian children can ranges from $8 \%$ to $22 \%$, with a greater prevalence of the Unilateral Cross Bite (UCB) [1,2]. This condition is characterised by an inverse relationship of the upper and lower buccal dental cusps and may involve one or several teeth [3].

Clinically the CB generates a mandibular deviation and an aesthetic decrease with a modification of facial midline [4]. The pathogenesis of this type of malocclusion is still unknown. The alteration of teeth contact may create in child an asymmetric muscles function [5] and an alteration of facial growth [6].

Many authors suggest that the CB is a condition related to a reduction of maxillary transversal growth with a mandibular displacement [7].

The most important evaluation of this type of malocclusion is the cephalometric postero-anterior analysis. This type of evaluation allows to have correct information about the maxillary and mandibular transversal growth [8].

Treatment of CB in growing patients has often made with Rapid Maxillary Expansion (RME) [9]. RME act by applying heavy intermittent force to split the midpalatal suture [10].

The effect in third part of face is a correction of the skeletal transverse dimension and the opening of nasal airway structure $[11,12]$. The expansion regime is a quarter turn $(0.25 \mathrm{~mm})$ twice a day [10] until the palatal cusps of upper molars occluded with the buccal cusp tips of lower molars [13]. Sometime the home made activation decrease the effect of the RME. Many studies evaluate the possible effect of RME on adults [14]. The treatment protocol, on young adults, provide the use of miniscrew in palatal side [15] or a Surgical Assisted Rapid Maxillary Expansion (SARME) [16].

In the present paper authors evaluated a new method of maxillary expansion: Nitanium ${ }^{\circledR}$ Palatal Expander TM (NPE) (Ortho Organizers, Inc. - San Marcos, CA). The NPE is a nocompliance fixed-removable orthodontic device which presents a central nickel-titanium alloy loop. The aim of the study was to evaluate the skeletal and dento-alveolar effects that NPE- 2 allows to obtain on the transverse plane, verifyng its effectiveness on permanent dentition in adults during the treatment of bilateral or unilateral posterior cross bite.

The shape memory is the ability that allows the device to switch back to the original form even after a deformation. In fact the metallic alloy of which it is composed can change its own characteristic by changing temperature $[17,18]$.

Considering that the transition temperature is around to $36^{\circ} \mathrm{C}$, below this temperature the interatomic forces become 
weak to the point that alloy is flexible and deformable, permitting an easy placement in the oral cavity. Above this temperature the alloy recovers rigidity and the appliance switches back to its original shape.

The stainless omega loops are connected to the primary portion of the Nitanium $\AA$ appliance, ending in two lingual tubes which fit into special lingual tubes welded to the bands cemented on the upper first molars. The omega loops are the only components of the apparatus that can be activated. Omega loops are connected to the arms in ortholoy of 0.036 inches in diameter that extends mesially and that can be modeled according to different orthodontic needs.

Very important is the identification of the correct size during the choice of the appliance that must also consider the thickness of bands and lingual tubes, as well as the objectives of transverse expansion [19].

The nickel-titanium expander, by expressing continuous and slight forces, determines a slow expansion of the upper jaw with a physiological adaptiation of the median palatine suture by affixing bone [20]. These results are comparable to those obtained with other devices that determine a slow expansion with evidence of separation of the median palatine suture radiologically detected in cases from $50 \%$ to $80 \%[21,22,23]$.

The appliance is able to determine, in a relatively short time and in every case without any cooperation of the patient, a derotation movement of molars on which it is applied, followed by an expansion movement [19]. The expansion is caused by a combination of opening on the median palatine suture and tipping of the dento-alveolar processes [19].

\section{Methods}

Twenty-one patients (13 females and 8 males, mean age 17.2 years) in permanent dentition with bilateral or unilateral posterior cross bite and a reduction of the transversal growth of the maxilla were selected. The participants and parents provided written informed consent to be involved in the study.

\section{Inclusion Criteria Included}

Hypodivergent skeletal pattern (NSL/ML $<30^{\circ}$; ANB $\geq 3.5^{\circ}$; overjet $\geq 4 \mathrm{~mm}$ ) and permanent dentition. Exclusion criteria included: oral or systemic diseases; missing teeth; congenital malformations and previous orthodontic treatment. 5 patients presented a bilateral cross bite and 16 patients presented an unilateral cross bite.

All patients were treated with Nitanium ${ }^{\circledR}$ Palatal Expander 2TM (NPE-2) applied to bands cemented with glassionomer cement (GC Fuji Ortho LC-GC Corporation) to the upper first molars. The NPE-2 has a palatal termo actived bar $\left(36^{\circ} \mathrm{C}\right)$ with wo elgiloy arms $[17,18]$.The NPE-2 selection was done as follow: [dSPS $-3 \mathrm{~mm}]+[\mathrm{dFCI}-\mathrm{dMPS}]$.

This formula explains the correct expansion required in NPE2 appliance. It is calculated on the distance between the upper first molars palatal surfaces (considering the cervical surfaces and the middle of the palatal surfaces in an antero-posterior plane - dSPS). The upper inter-molar distance is obtained subtracting 3 $\mathrm{mm}$, which represents the bands and tubes thickness. All this is added on the difference between the distances dMPS (distance of mesio-palatal cusp) and dFCI (distance between the lower molars central fossa) develops, and it represents the transverse defect value between the two arches [20].

Pre-treatment and post-treatment records included maxillary and mandibular casts, photographs, panoramic radiograph, occlusal radiograph, lateral and postero-anterior radiographs (Kodak 8000C). Head films were taken with the patient fixed in a cephalostat, in centric occlusion, with adequate visualization of reference structures and no appreciable rotation of the head. Cephalometric analyses were performed before phase 1 treatment (T1), and immediately following phase 2 treatment (T2). To reduce the error of the method, cephalometric radiographs were selected randomly and reanalyzed 30 days later by the same examiner.

On lateral radiographs was performed a cephalometric tracing according to the standard method while on posteroanterior film the cephalogram was performed according to Giannì method [24] Figure 1.

On this particular cephalogram parameters such as the distance between the maxillary points right and left (Mxd - Mxs), between left and right upper molars points $(\mathrm{CVM}+\mathrm{d}-\mathrm{CVM}+\mathrm{s})$, between the right and left occlusal points (OCCLd - OCCLs) and between left and right lower molars points (CVM-d - CVM-s) were evaluated Table 1.

On plaster models, instead, some distances were estimated by using a Vernier scale Table 2: intermolar distance $(6+6)$ measured at centroid [25]; interpremolar distance measured at the top of the central fossa of the first premolar (4+4); upper intercanine distance between the cusps of the canines $(3+3)$; the perimeter of the upper arch measured between the mesial contact points of the upper first molars along the dental arch; the lower intermolar distance (6-6) detected at the level of the central fossa of the lower molars; the intercanine lower distance (3-3) between the cusps of the canines.

Each patient was subjected to control visits every 3 weeks in order to follow the progress of the expansion Figures 2,3,4,5,6.

In 5 out of 21 cases an activation of the steel omega loops was operated to achieve a further dentoalveolar expansion, while in one case only the opposite operation was carried out because of a buccal tipping of the maxillary first molars.

\section{Statistical analysis}

A statistical analysis of cephalometric values at the beginning of treatment (T1) and at six months after the application of the device (T2) were detected. Data were evaluated on statistical "GraphPad" software performing a Paired t-test. Statistical significance was set at 0.05 . After noting the indicated 


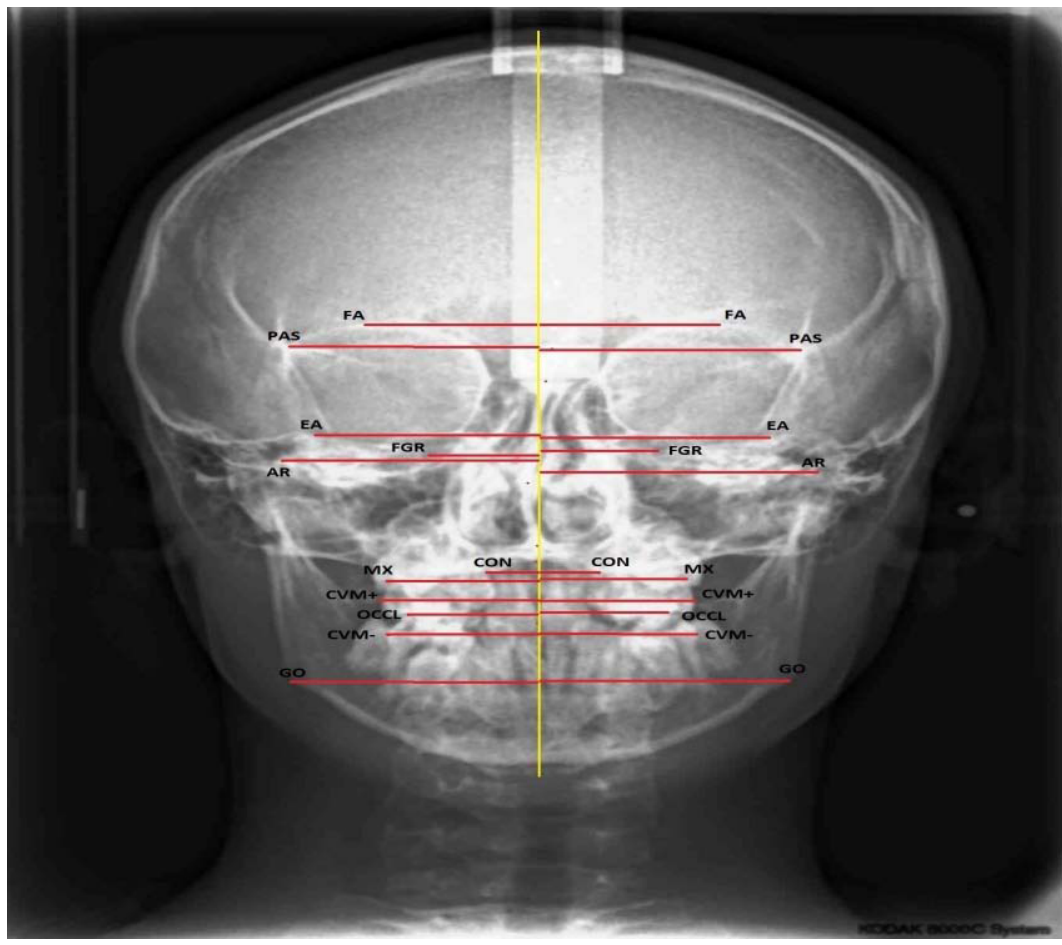

Figure 1: Posteroanterior cephalogram indicating identification of landmarks and linear measurements according to Giannì. Landmarks - FA: higher orbital point; PAS: orbitosphenoidal point - cephalometric point of coincidence of the anterior margin of the lesser wing of the sphenoid bone and the upper margin of the greater wing of the sphenoid bone with the superior-lateral margin of the orbit; EA: arcuate eminence of the petrous; FGR: geometric center of the large round hole; AR: articular point - highest point of the head of the condyle in the horizontal plane; CON: occipital point cephalometric point of coincidence between lower end of the condyle of the occipital bone and contour of the great occipital foramen; MX: maxillary point - crossing point of the concavity of the zygomatic process of the maxilla with the line of the tuber; $\mathrm{CVM}+$ : maxillary point - most prominent point on the sagittal plane of the buccal surface of the upper first molar; OCCL: occlusal point; CVM-: lower molar point - most prominent point on the sagittal plane of the buccal surface of the lower first molar; GO: gonion - lateral and inferior border of the mandibular angle.

Table 1: Linear measurements (in $\mathrm{mm}$ ) recorded on postero-anterior cephalogram according to Giannì.

\begin{tabular}{|c|c|c|c|c|c|c|c|}
\hline \multicolumn{2}{|c|}{} & \multicolumn{2}{|c|}{ T1 } & \multicolumn{2}{c|}{ T2 } & D & P \\
\hline \multicolumn{1}{|c|}{ Parameters } & Average & St. dev & Average & St. dev & T1-T2 & T1-T2 \\
\hline $\mathbf{1}$ & Mxd-Mxs & 62.7 & 2.23 & 63.9 & 1.88 & 1.2 & $*$ \\
\hline $\mathbf{3}$ & CVM+d - CVM+s & 57.2 & 1.78 & 60.1 & 1.67 & 2.9 & $*$ \\
\hline $\mathbf{4}$ & OCCLd - OCCLs & 48.4 & 2.02 & 50.3 & 2.46 & 1.9 & $*$ \\
\hline
\end{tabular}

Table 2: Measurements made on casts (in $\mathrm{mm}$ ) at T1 and T2.

\begin{tabular}{|c|c|c|c|c|c|c|c|}
\hline \multirow{2}{*}{\multicolumn{2}{|c|}{ Parameters }} & \multicolumn{2}{|c|}{ T1 } & \multicolumn{2}{|c|}{$\mathbf{T} 2$} & \multirow{2}{*}{$\begin{array}{c}\text { D } \\
\text { T1-T2 }\end{array}$} & \multirow{2}{*}{$\begin{array}{c}\mathrm{P} \\
\text { T1-T2 }\end{array}$} \\
\hline & & Average & St. dev & Average & St.dev & & \\
\hline 1 & $6+6$ & 44.4 & 1.86 & 46.7 & 1.76 & 2.3 & $* *$ \\
\hline 2 & $4+4$ & 36.2 & 2.20 & 37.8 & 2.28 & 1.6 & $* *$ \\
\hline 3 & $3+3$ & 33.4 & 3.26 & 34.8 & 3.01 & 1.4 & $*$ \\
\hline 4 & Arch perim. & 78.1 & 3.32 & 79.6 & 3.28 & 1.5 & NSS \\
\hline 5 & 6-6 & 39.8 & 1.23 & 41.2 & 1.46 & 1.4 & NSS \\
\hline 6 & 3-3 & 28.2 & 1.12 & 28.8 & 1.44 & 0.6 & NSS \\
\hline
\end{tabular}

Citation: Montaruli G, Laurenziello M, Lo Russo L, Chimenti C, Lo Muzio L, et al. (2016) Bilateral or Unilateral Cross-Bite Treatment with Nickel Titanium Palatal Expander (NPE-2) in Young Adults J Dent Oral Disord Ther 4(2): 1-6. 


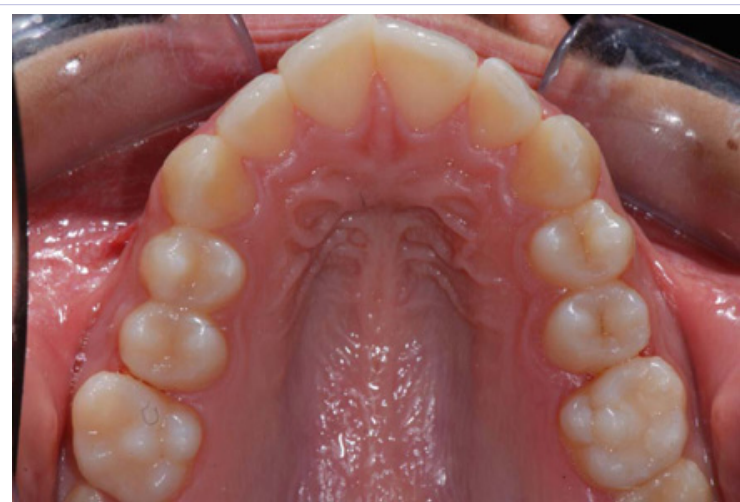

Figure 2: Occlusal view of a case of transverse contraction associated with unilateral posterior cross bite in adult.

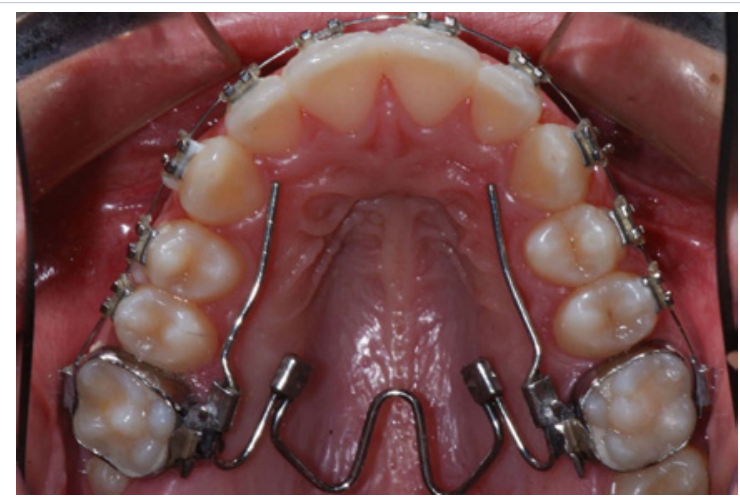

Figure 3: The Nitanium ${ }^{\circledR}$ Palatal Expander $2^{\mathrm{TM}}$ is a fixed-removable orthodontic appliance whose active part is the central nitanium alloy loop. The arms in ortholoy requires periodic adjustments. If not needed they can be removed.

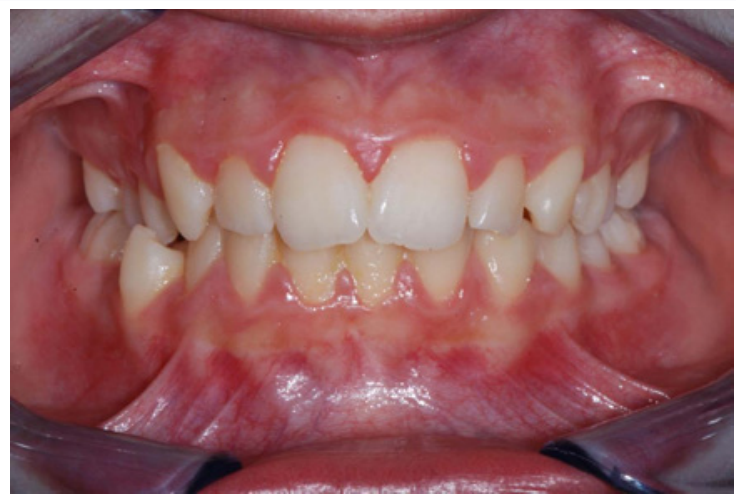

Figure 4: Intraoral frontal view of a case of unilateral posterior crossbite in adult before the placement of NPE-2.

measurements, averages and standard deviations were calculated for each parameter identified.

\section{Results}

The tables show the averages and standard deviations at $\mathrm{T} 1$ and $\mathrm{T} 2$ of the parameters measured on the posteroanterior cephalogram (Table 1) and on the casts (Table 2). The differences between $\mathrm{T} 1$ and $\mathrm{T} 2$, as well as the level of significance of the changes obtained with the expansion by Nitanium ${ }^{\circledR}$ Palatal Expander 2TM. At the end of the expansion phase a significant average increase $(P<0.01)$ of $2.9 \mathrm{~mm}$ between right and left upper molars points $(\mathrm{CVM}+\mathrm{d}-\mathrm{CVM}+\mathrm{s})$ was detected on posteroanterior cephalometric tracing. Also the average increase of the distances between the right and left occlusal points (OCCLd - OCCLs) of $1.9 \mathrm{~mm}(P<0.05)$ and of $1.2 \mathrm{~mm}(P<0.05)$ between the maxillary right and left points (Mxd - Mxs) were significant.

The average increase of the distance between left and right lower molars points (CVM-d - CVM-s) resulted not statistically significant.

The measurements on casts showed a significant increase $(P$ $<0.01$ ) on the transverse plane of the upper intermolar distance $(6+6)$ of $2.3 \mathrm{~mm}$ and of the upper intercanine distance (3+3) of 1.6 $\mathrm{mm}$. A statistically significant increase $(P<0.05)$ of the average upper interpremolar distance $(4+4)$ of $1.4 \mathrm{~mm}$ was detected. The average increases of the arch perimeter, of the lower intermolar (6-6) and lower intercanine distances (3-3) were not statistically significant. No evidence of radiographic radiolucency at the median-palatal suture level was detected in treated patients.

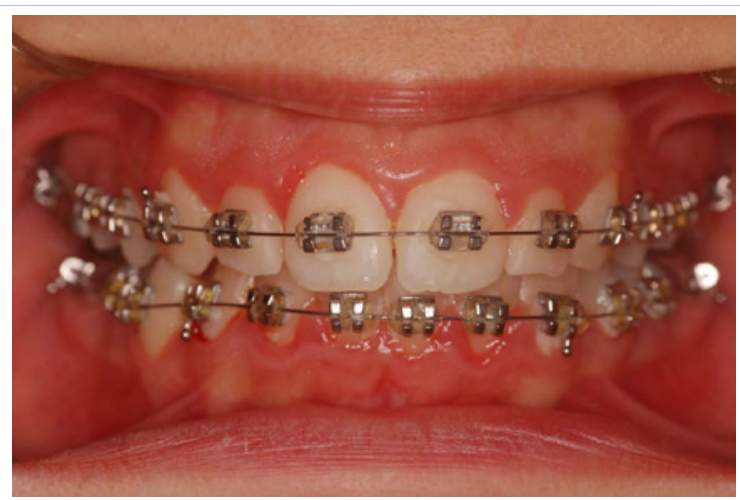

Figure 5: Intraoral frontal view of the case 6 months after the placement of NPE-2.

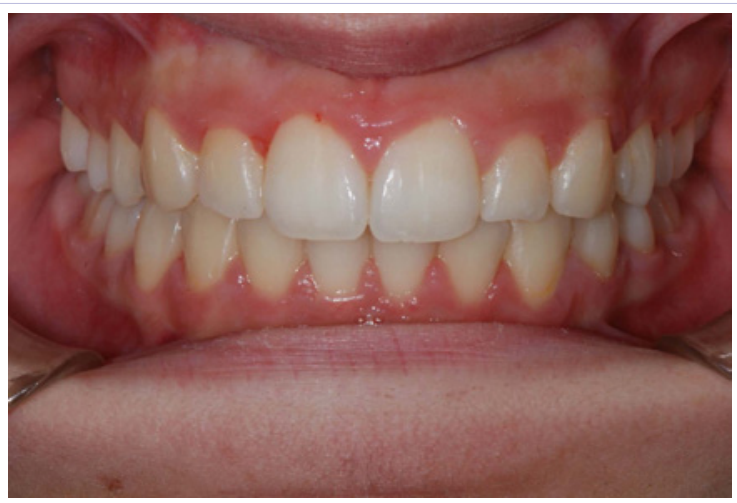

Figure 6: Intraoral frontal view of the case immediately after the removal of NPE-2. 


\section{Discussion}

The treatment options of the posterior cross bite in young adults are: rapid maxillary expansion [26,27], rapid maxillary expansion with miniscrew [15] and Surgical Assisted Rapid Maxillary Expansion (SARME) [28].

The RME is done with fixed appliance on upper molars. The activation on transversal plane may be done with a palatal screw (RME), or with the use of Slow Maxillary Expansion (SME) such as Quad-elix or Goshgarian arch [29,30].

The most common risk in adults use of conventional RME such as expansion failure or limited skeletal expansion, instability of results, pain, tissue swelling, buccal crown tipping, gingival recession, root resorption and ulceration have been reported [31]. In the last years the SARME method has been frequenly used to overcome the mentioned limitation of RME. Another treatment option was to use in association with the RME appliance mini screws in palatal bone (MARME: miniscrew assisted RME) [15]. This type of treatment had greater orthopedic effects in adolescents than young adults where the midpalatale suture is closed [32,33]. The above mentioned technique (SARME and MARME) require a surgical procedures and sometimes patients refuse this type of approach [15]. Other type of fixed oral appliance used in adults treatment are the quad helix [29].

A new no compliance method to treat the maxillary transversal alteration is the NPE-2 [17]. This type of oral appliance is a slow maxillary expander used with fixed appliance for teeth alignment [34]. The NPE-2 produce a continuous light pressure on the teeth and requires little patient cooperation and laboratory work. Another important aspect about the NPE-2 use is the upper molars rotation and distalization that was observed during its use [17]. In the present paper the data collected on the dental points, as verified on casts, and on the bone, as verified on posteroanterior cephalograms, underline how the NPE-2 is able to obtain significant dental and slight skeletal results.

In the present study the NPE-2 showed a maxillary expansion in young adults of $1.2 \mathrm{~mm}(P<0.05)$. These result is in agreement with other studies $[35,19]$ is which is shown how the most important effect NPE was a controlled dentoalveolar expansion and a slight bone remodeling. As suggested in many papers the most important effect of the NPE was on molar transversal position [20]. Ciambotti, et al. showed an increase in the intermolar width of $6.26 \mathrm{~mm}$ in patients with NPE [35]; Donohue, et al. evaluated an increase of upper molars distance of $7.8 \mathrm{~mm}$ and $5.9 \mathrm{~mm}$ on first molars [36].

Infact the present study the NPE-2 generated an effective vestibular position of upper molars of $2.3 \mathrm{~mm}(P<0.01)$ on casts and $2.9 \mathrm{~mm}(P<0.01)$ on headfilm. This difference was related to the different point of measurement: on casts was measured on palatal part of the upper molar while on headfilm was measured on vestibular part of the upper molars. Authors showed how no important modification was observed on lower molars both on cephalometric evaluation $(0.8 \mathrm{~mm}$; NS) that on casts $(1.4 \mathrm{~mm}$;
NS). A teeth expansion was observed on first premolars $(1.6 \mathrm{~mm}$; $P<0.01)$ and on canines $(1.4 \mathrm{~mm} ; P<0.01)$.

\section{Conclusion}

The most important effects of the NPE-2 were:

- maxillary molars expansion

- $\quad$ small expansion of the maxilla

- no modifications on lower molars position

The dentoalveolar changes induced by the application of NPE- 2 and the correction of bilateral or unilateral CB showed the effectiveness of this device even in adults in permanent dentition. The NPE- 2 is a no compliance device and requires a limited number of controls and minimal clinician intervention. It develops continuous and slow forces and it is programmed to achieve the request expansion entity.

The benefits of a thermo activated system were about the possibility of change the appliance activation; the easy management of appliance for clinicians and pain control for patients.

\section{Authors Contributions}

Graziano Montaruli and Domenico Ciavarella carried out the study and drafted the manuscript. Michele Laurenziello provided statistical support. Lucio Lo Russo participated in the design of the study and analyzed the data. Claudio Chimenti and Lorenzo Lo Muzio designed and coordinated the study. All authors read and approved the final manuscript.

\section{Competing Interests}

The authors declare that they have no competing interests.

\section{References}

1. Egermark-Eriksson I, Carlsson GE, Magnusson T, Thilander B. A longitudinal study on malocclusion in relation to signs and symptoms of cranio-mandibular disorders in children and adolescents. Eur J Orthod. 1990;12(4):399-407.

2. Airoldi G, Riva G, Vanelli M, Filippi V, Garattini G. Oral environment temperature changes induced by cold/hot liquid intake. Am J Orthod Dentofacial Orthop. 1997;112(1):58-63.

3. Bourke, A, Daskalogiannakis J, Tompson B, Watson P. Force characteristics of nickel-titanium open-coil springs. Am J Orthod Dentofacial Orthop. 2010;138(2):142e141-147; discussion 142-143. doi: 10.1016/j.ajodo.2010.01.026.

4. M. Thomas Graber, Thomas Rakosi, Alexandre G. Petrovic. Ortopedia dentofacciale con dispositivi funzionali. Elsevier, 1998.

5. Ciavarella D, Antonio Palazzo, Alfredo De Lillo, Lucio Lo Russo, Sergio Paduano, Luigi Laino, et al. Influence of vision on masticatory muscles function: surface electromyographic evaluation. Ann Stomatol (Roma). 2014;5(2):61-65.

6. G. Montaruli, C. Caradonna, M. Castellucci, E. Ciccarelli. Functional treatment of mandibular lateral deviation. Trattamento funzionale delle latero-deviazioni mandibolari. Mondo Ortod. 2010;35(3):128139. 
7. Lindner A, Henrikson CO, Odenrick L, Modéer T. Maxillary expansion of unilateral cross-bite in preschool children. Scand J Dent Res. 1986;94(5):411-418.

8. Tateishi C, Moriyama K, Takano-Yamamoto T. Dentocraniofacial morphology of 12 Japanese subjects with unilateral cleft lip and palate with a severe Class III malocclusion: a cephalometric study at the pretreatment stage of surgical orthodontic treatment. Cleft Palate Craniofac J. 2001;38(6):597-605.

9. Kilic N, Kiki A, Oktay H, Erdem A. Effects of rapid maxillary expansion on Holdaway soft tissue measurements. Eur J Orthod. 2008;30(3):239243.

10. Bishara SE and RN Staley. Maxillary expansion: clinical implications. Am J Orthod Dentofacial Orthop. 1987;91(1):3-14.

11. Timms DJ. The reduction of nasal airway resistance by rapid maxillary expansion and its effect on respiratory disease. J Laryngol Otol. 1984;98(4):357-362

12. Doruk C, Sökücü O, Sezer H, Canbay EI. Evaluation of nasal airway resistance during rapid maxillary expansion using acoustic rhinometry. Eur J Orthod. 2004;26(4):397-401.

13. Ugolini A, Cerruto C, Di Vece L, Ghislanzoni LH, Sforza C, Doldo T. Dental arch response to Haas-type rapid maxillary expansion anchored to deciduous vs permanent molars: A multicentric randomized controlled trial. Angle Orthod. 2015;85(4):570-576.

14. Handelman CS, Wang L, BeGole EA, Haas AJ. Nonsurgical rapid maxillary expansion in adults: report on 47 cases using the Haas expander. Angle Orthod. 2000;70(2):129-144.

15. Choi SH, Shi KK, Cha JY, Park YC, Lee KJ. Nonsurgical miniscrewassisted rapid maxillary expansion results in acceptable stability in young adults. Angle Orthod. 2016.

16. Seeberger R, Gander E, Hoffmann J, Engel M. Surgical management of cross-bites in orthognathic surgery: Surgically assisted rapid maxillary expansion (SARME) versus two-piece maxilla. J Craniomaxillofac Surg. 2015;43(7):1109-1112.

17. Arndt WV. Nickel titanium palatal expander. J Clin Orthod. 1993;27(3):129-137

18. Caniklioglu MC. Use of a nickel titanium palatal expander in cleftpalate cases. J Clin Orthod. 2004;38(7):374-377.

19. Ferrario VF, Garattini G, Colombo A, Filippi V, Pozzoli S, Sforza C. Quantitative effects of a nickel-titanium palatal expander on skeletal and dental structures in the primary and mixed dentition: a preliminary study. Eur J Orthod. 2003;25(4):401-410.

20. Karaman AI. The effects of nitanium maxillary expander appliances on dentofacial structures. Angle Orthod. 2002;72(4):344-354

21. Pinheiro FH, Daniela Gamba Garib, Guilherme Janson, Roberto Bombonatti, Marcos Roberto de Freitas. Longitudinal stability of rapid and slow maxillary expansion. Dental Press J Orthod. 2014;19(6):7077.

22. de Medeiros Alves AC, Garib DG, Janson G, de Almeida AM, Calil LR.
Analysis of the dentoalveolar effects of slow and rapid maxillary expansion in complete bilateral cleft lip and palate patients: a randomized clinical trial. Clin Oral Investig. 2015;

23. Esenlik E. and M. Rubenduz. An evaluation of the dentoskeletal effects of slow maxillary expansion from the mixed to the permanent dentition. Aust Orthod J. 2015;31(1):2-13.

24. Farronato G, Maspero C, Esposito L, Briguglio E, Farronato D, Giannini L. Rapid maxillary expansion in growing patients. Hyrax versus transverse sagittal maxillary expander: a cephalometric investigation. Eur J Orthod. 2011;33(2):185-189.

25. Chang JY, McNamara JA Jr, Herberger TA. A longitudinal study of skeletal side effects induced by rapid maxillary expansion. Am J Orthod Dentofacial Orthop. 1997;112(3):330-337.

26. Sakai $\mathrm{T}$ and H.Hanaoka. Study on maxillary rapid expansion method. II. Changes of eight cases (author's transl). Nihon Kyosei Shika Gakkai Zasshi. 1978;37(3):306-314.

27. Sorel 0. Rapid palatal expansion for the treatment of maxillary constriction. Rev Stomatol Chir Maxillofac. 2004;105(1):26-36.

28. Altug Atac AT, Karasu HA, Aytac D. Surgically assisted rapid maxillary expansion compared with orthopedic rapid maxillary expansion. Angle Orthod. 2006;76(3):353-359.

29. Hicks EP. Slow maxillary expansion. A clinical study of the skeletal versus dental response to low-magnitude force. Am J Orthod. 1978;73(2):121-141.

30. Frank SW and GA Engel. The effects of maxillary quad-helix appliance expansion on cephalometric measurements in growing orthodontic patients. Am J Orthod. 1982;81(5):378-389.

31. Cao Y, Zhou Y, Song Y, Vanarsdall RL Jr. Cephalometric study of slow maxillary expansion in adults. Am J Orthod Dentofacial Orthop. 2009;136(3):348-354.

32. Park HS. A miniscrew-assisted transpalatal arch for use in lingual orthodontics. J Clin Orthod. 2006;40(1):12-16.

33. Lee KJ, Park YC, Park JY, Hwang WS. Miniscrew-assisted nonsurgical palatal expansion before orthognathic surgery for a patient with severe mandibular prognathism. Am J Orthod Dentofacial Orthop. 2010;137(6):830-839.

34. Henry RJ. Slow maxillary expansion: a review of quad-helix therapy during the transitional dentition. ASDC J Dent Child. 1993;60(4):408413.

35. Ciambotti C, Ngan P, Durkee M, Kohli K, Kim H. A comparison of dental and dentoalveolar changes between rapid palatal expansion and nickel-titanium palatal expansion appliances. Am J Orthod Dentofacial Orthop. 2001;119(1):11-20.

36. Donohue VE, Marshman LA, Winchester LJ. A clinical comparison of the quadhelix appliance and the nickel titanium (tandem loop) palatal expander: a preliminary, prospective investigation. Eur J Orthod. 2004;26(4):411-420. 